\title{
Non-Darcy Skin Effect with a New Boundary Condition
}

\author{
Fahd Siddiqui", Mohamed Y. Soliman
}

University of Houston, 5000 Gulf Freeway, USA,

\begin{abstract}
Use of numerical methods has been a popular tool for engineers especially with the advent of faster computers. This study uses the finite element method to solve the non-Darcy flow problem with a new boundary condition involving step functions.

The problem of non-Darcy skin reconsidered in terms of a distinct skin value during production and decreasing to a smaller value during well shut-in. The boundary condition is formulated and solution is performed using commercial finite element and finite difference solvers/simulators. We show that the current prevalent model of using non-Darcy coefficient to model this changing skin behavior is adequate for engineering purposes.
\end{abstract}

Keywords: Non-Newtonian Fluids; Wellbore Storage; Fluid Flow through Porous Media

\section{INTRODUCTION}

Darcy's Law sufficiently describes the flow of Newtonian fluids in porous media. Darcy's Law is strictly valid for viscous flow (i.e. $\mathrm{Re} \rightarrow 0$ ). It is generally used for modeling flow in petroleum reservoirs and aquifers, because the low-matrix permeability results in low velocities. At low velocities, Siddiqui et al. (Siddiqui, Soliman, Waylon, \& Akif, 2016) have already showed a deviation of Darcy's law which prevails away from the wellbore. However, higher velocities are often observed in fractures and near wellbores; a more complicated model is needed to describe flow in these cases.

Forchheimer's equation (Forchheimer, Wasserbewegung durch Boden, 1901) is an extension to Darcy's Law and models the nonlinearities that occur because of inertial effects:

$-\nabla p=\frac{\mu}{k} v+\rho \beta v^{2}$

The constant, $\beta$, is referred to as the non-Darcy coefficient and is found experimentally. It represents the additional inertial resistance caused by the converging/diverging and tortuous medium geometry. A bundle of straight tubes have a non-Darcy coefficient of exactly zero. The above equation reduces to Darcy's law for small values of velocities.

Forchheimer's equation fits some experimental data very well by Forchheimer $(1901 ; 1930)$ and others (Blake, 1922; Fancher, Lewis, \& Barnes, 1932; Lindquist, 1933; Brownell, Gami, Miller, \& Nekarvis, 1956; Mobasheri \& Todd, 1963; Sunada, 1965; Ahmed, 1967; Kim, 1985). However, the equation does not match other experimental data (Forchheimer, 1930; Barree \& Conway, 2004; Barree \& Conway, 2005). Even in 1901 Forchheimer conceded this fact an added a cubic term to fit the experimental data that would otherwise not fit Equation Error! Reference source not found..

Barree and Conway $(2004 ; 2005)$ conducted experiments and produced data that did not follow Equation Error! Reference source not found., suggesting that Forchheimer's equation is not valid over a large range of velocities. The arguments made by Barree and Conway (2004; 2005) for a minimum-permeability plateau has validity and are supported theoretically and numerically.

In their paper, Barree and Conway (2005) also suggested permeability obtained by extrapolation to the intercept in a Forchheimer plot is not the Darcy permeability. Figure 1 shows their findings that a Forchheimer straight line is not observed from measure data. If an apparent Darcy permeability is measured at low rate and the apparent $\beta$ is measured from the tangent to the data at high rate, the actual flow capacity of the system will be misrepresented by Forchheimer's Equation (lower line). If the consistent slope and intercept of the high-rate data is used, the flow capacity will be severely underestimated at low rate (upper line). Therefore, they concluded that only a continuously changing apparent flow capacity will accurately describe the system at all flow rates. 


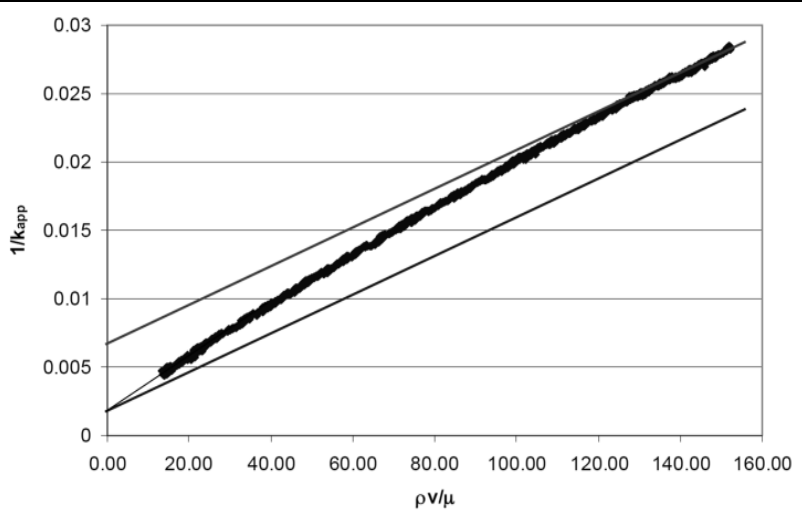

Figure1. Deviation from Forchheimer's Linear Equation (Barree \& Conway, 2005)

Therefore they suggested a Logistic Dose model for describing the non-linearity, which is given by Equations Error! Reference source not found., Error! Reference source not found.andError! Reference source not found.. If $k_{\min }$ is set to zero, the Forchheimer's original linear equation is obtained. Barree and Conway (2004) describe a procedure with which the values of parameters $k_{\min }, k$ ${ }_{d}$ and $\tau_{l}$ can be obtained to from conventional experiments conducted at various velocities.

$$
\begin{aligned}
& \nabla p=\frac{\mu v}{k_{d}}\left(1+\frac{\beta k_{d} \rho v}{\mu}\right) \\
& k_{\text {app }}=k_{\min }+\frac{\left(k_{d}-k_{\text {min }}\right)}{\left(1+R_{e}^{F}\right)^{E}} \\
& R_{e}=\frac{\rho v}{\mu \tau_{l}}
\end{aligned}
$$

\section{NON-DARCY Flow COEFFicient CORRELATIONS}

Non-Darcy coefficient $\beta$ correlations are functions of permeability, porosity and sometimes tortuosity. $\beta$ factor is the degree of tortuosity of porous channels related to particle acceleration. Empirical correlations have been suggested by many researchers, some of the more popular ones are listed below. However, finding $\beta$ is most commonly accomplished by experiments or pressure transient tests. Equations Error! Reference source not found., Error! Reference source not found.andError! Reference source not found. assume permeability in $m D$ and $\beta$ in $1 / f t$.

Geertsma (1974) suggested a model based on dimensional analysis, experimental data and physical considerations shown in Equation Error! Reference source not found.. The author prescribes the phenomena to laminar flow regime and the deviations to be purely due to acceleration of the fluid in pore spaces.

$\beta=\frac{4.85 \times 10^{4}}{\phi^{5.5} k^{0.5}}$

Jones (1987) studied the influence of permeability heterogeneity on non-Darcy coefficient on 355 sandstone and 29 limestone cores. The final relationship from his analysis is given in Equation Error! Reference source not found..

$$
\beta=\frac{6.15 \times 10^{10}}{k^{1.55}}
$$

Pascal, Ronald and Kingston (1980) suggested a mathematical model to estimate the fracture length and the non-Darcy coefficient from variable flow drawdown tests of shallow, low permeability gas reservoirs. Equation Error! Reference source not found. gives the final form of the developed relationship.

$\beta=\frac{1.46 \times 10^{12}}{k^{1.176}}$ 
An extensive collection of correlations can be found in the published papers (Frederick Jr. \& Graves, 1994; Li \& Engler, 2001).

\subsection{Problem Analysis}

The diffusivity equation generally used in the literature is based off of Darcy's Law. However, as discussed, Darcy's law may not be valid for high velocity flows especially in the near wellbore region or in fractured reservoirs. Wattenbarger and Ramey (1968) derived and solved the differential equation considering the turbulent flow during the flow period. Equation Error! Reference source not found. gives the solution in terms of pseudo-pressure in field units.

$\psi_{w f}=\psi_{i}+\frac{1.637 q_{g} T}{k h}\left[\log \left(\frac{1688 \phi \mu_{g} c_{t}}{k t_{p}}\right)+\frac{\left(s+D q_{g}\right)}{1.151}\right]$

The authors approximated the effect of turbulence by an additional skin drop $\left(D q_{g}\right)$ in the equation. Soliman et al. (1991) had claimed that this approximation is valid as long as turbulent flow regime does not prevail through a large portion of the reservoir. That is, approximating the turbulence effect for tests with a radius of investigation of more than 50ft will yield an acceptable accuracy from approximating the turbulence effect with an additional rate dependent skin term $(D)$.

This study uses the novel framework suggested by Soliman et al. (1991) to model the post-Darcy turbulence effect with different skin values during drawdown and buildup. The authors had used unit step functions to model the problem of changing skin as the well is shut-in.

This study will use numerical simulators to examine this effect of changing skin and shows that indeed it is correct from an engineering point of view to model non-Darcy turbulence equivalently as a problem of changing skin.

\subsection{Numerical Simulation}

Numerical tools have become a popular choice for engineering calculations, and this study uses and relies on the robustness of two such different numerical tools: Finite Element and Finite Difference. For finite element method, a powerful software, COMSOL Multiphysics was used to solve the governing partial differential equation. For finite difference, QuikLook simulator was used, which is a petroleum industry standard software for reservoir simulation.

\section{Finite Element MOdel With COMSOL}

COMSOL was used to solve the differential equations for changing skin problem. The problem was defined by the Equations Error! Reference source not found.throughError! Reference source not found..

$$
\begin{aligned}
& \frac{\partial^{2} p_{D}}{\partial r_{D}^{2}}+\frac{1}{r_{D}} \frac{\partial p_{D}}{\partial r_{D}}=\frac{\partial p_{D}}{\partial t_{D}} \\
& p_{D}\left(r_{D}, t_{D}=0\right)=0 \\
& p_{D}\left(r_{D} \rightarrow \infty, t_{D}\right)=0 \\
& C_{D} \frac{\partial p_{w D}}{\partial t_{D}}-\left.\frac{\partial p_{D}}{\partial r_{D}}\right|_{r_{D}=1}=1-U\left(t_{D}-t_{p D}\right) \\
& p_{w D}=p_{D}-\left.\left[1-U\left(t_{D}-t_{p D}\right) s_{D D}+U\left(t_{D}-t_{p D}\right) s_{B U}\right] \frac{\partial p_{D}}{\partial r_{D}}\right|_{r_{D}=1}
\end{aligned}
$$

Equation Error! Reference source not found.describes the governing partial differential equation of the flow of fluids through porous media. Equations Error! Reference source not found.andError! Reference source not found. describe the initial conditions, and the outer boundary condition of the problem. Equation Error! Reference source not found. uses the step function to impose the drawdown 
shut-in on the flow rate (inner boundary). It essential states that at time $t_{p D}$ and after, the dimensionless rate goes from 1 to 0 . What is novel about using step functions is described by the second part of the inner boundary condition in Equation Error! Reference source not found.. By using step functions, the equation imposes the condition of changing skin from skin during drawdown $\left(s_{D D}\right)$ to skin during buildup $\left(s_{B U}\right)$ at dimensionless time larger than $t_{p D}$. Figure 6.2 shows the step function used to model the rate change.

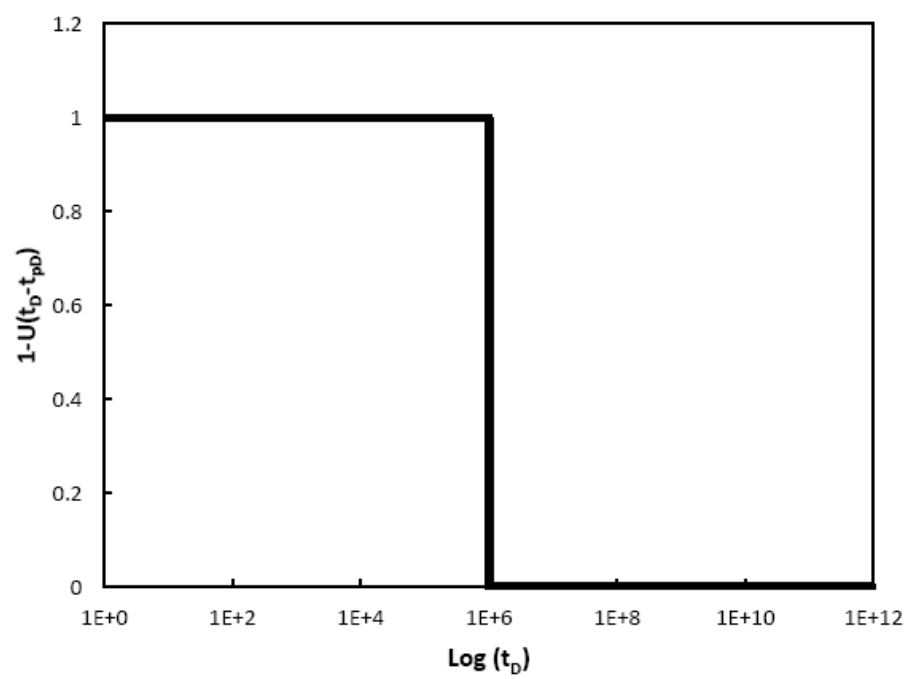

Figure2. Unit Step function

The above equations were input into COMSOL for solution using finite element method. The solver was run for various scenarios of $s_{D D}, s_{D D}$ and $C_{D}$. The software was run for dimensionless times up to $1 \times 10^{8}$. Automatic time stepping was used to choose time steps based on backward differentiation formula (BDF) varying between the order of 1 and 5.

Meshing was performed on an axis symmetric (radial) line interval with the left end point at $1\left(r_{D}=1\right)$ and right end point being sufficiently large to effectively become infinite acting $\left(r_{D} \rightarrow \infty\right)$. The size of the mesh was chosen to be increasing logarithmically increasing away from the wellbore, to avoid unnecessarily large number of mesh elements.

\subsection{COMSOL Results}

Results were generated for various realistic values of skin to determine the dimensionless pressure response. The dimensionless initial pressure was assumed to be 25 and the dimensionless producing time was taken at $1 \times 10^{6}$ Figure shows the resulting dimensionless pressure drop for $C_{D}=100$. The results reveal that the skin during buildup has no consequence on the pressure response during buildup, because the pressure responses coincide after shut-in, with the only difference being the last flowing pressures are lower for higher skin values and vice versa. The same trend result is obtained for a larger value of $C_{D}=1000$ in Figure.

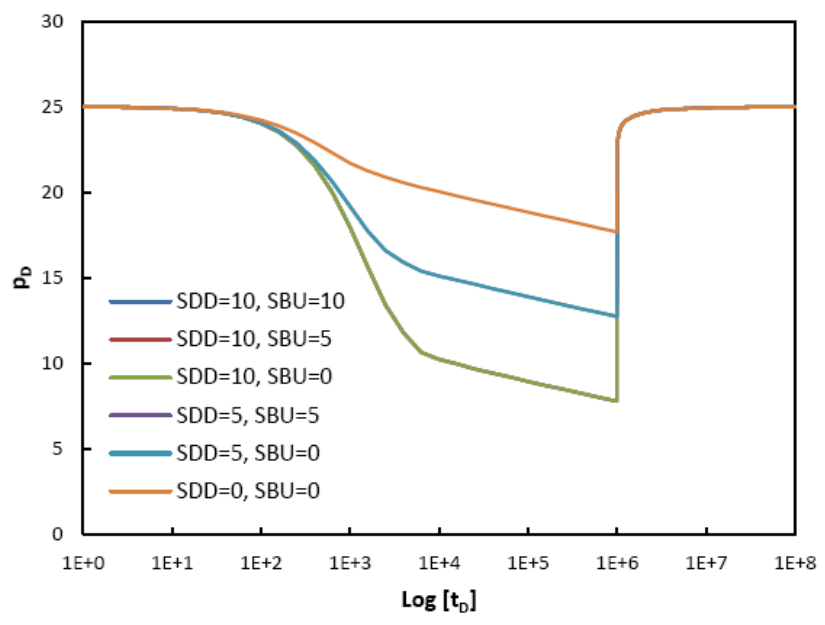

Figure3. Dimensionless Pressure Response for $C_{D}=100$ 


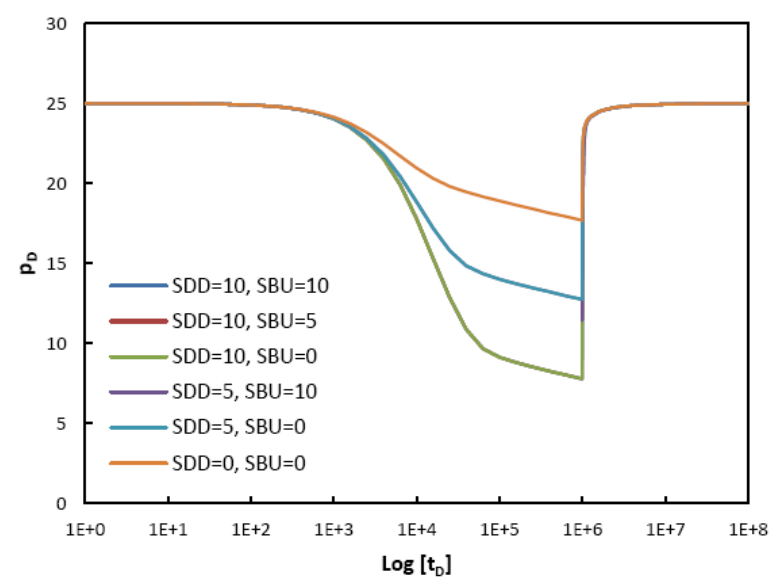

Figure4. Dimensionless Pressure Response for $C_{D}=1000$

It is well known that skin has no effect on the radial flow regime during buildup because skin appears in the inner boundary condition with the wellbore storage. And as soon as the pressure gradient (i.e. rate) vanishes during buildup, the effect of the skin goes away and radial flow (buildup) regime is manifested. However, the subtle point here is that superposition is still valid i.e. the non-linear equations described earlier soon become essentially linear paving the way for the validity of superposition. This will be explored in the next section with the reservoir simulator. This point is also supported by Figure which shows the Horner plot for various skin value scenarios. It shows that curves with the skin during drawdown of 10 build the pressure back up with the same rate without any effect from the skin during buildup; and similarly, the pressure response during buildup is not affected by the buildup skin for the case of skin during drawdown of 5. The small fluctuations in Figure are attributed to the numerical instability arising from the fact that step functions cause a drastic change in the condition which results in numerical challenges for the solver.

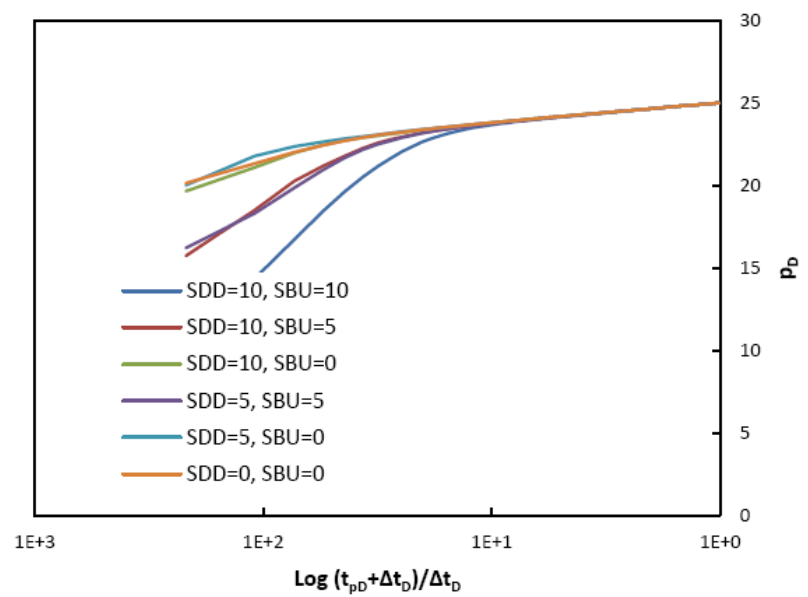

Figure5. Horner plot for various skin values for $C_{D}=1000$.

\section{RESERVOIR Simulation WITH QUiKLOOK}

One of the industry standard reservoir simulator, QuikLook was used to study the effect of $\beta$-factors on the pressure response and to show that the buildup pressure is unaffected by the $\beta$-factors except for the last flowing pressure (which is required to compute the skin).

The simulator was input with the reservoir and well properties given in Table. A radial grid with one well in the center was created. The well was setup to have a skin of 5 and a $\beta$-factor of $1 \times 10^{11} 1 / \mathrm{ft}$ and $1 \times 10^{14} 1 / \mathrm{ft}$ as separate cases. Another case was also setup in the simulator to have a skin of 5 during drawdown and 8.9 during buildup. This was done to approximately model the pressure response of $\beta=1 \times 10^{14} 1 / \mathrm{ft}$.

Figure shows the pressure vs time for the three cases. The results are in agreement with those obtained using COMSOL, i.e. the non-Darcy effect does not influence the pressure buildup response, but only the pressure drawdown response. Moreover the using skin to model the non-Darcy effect is an acceptable within engineering accuracy. 
Fahd Siddiqui \& Mohamed Y. Soliman

Table1. Reservoir and well properties

\begin{tabular}{|c|c|c|}
\hline$\varphi$ & 0.1 & $c p$ \\
\hline$\mu$ & 1 & $f t$ \\
\hline$r_{w}$ & 0.4 & $m d$ \\
\hline$k$ & 1 & $S t b / D$ \\
\hline$q$ & 100 & $f t$ \\
\hline$h$ & 50 & $h r s$ \\
\hline$t_{p}$ & 6 & $p s i$ \\
\hline$p_{i}$ & 5000 & $1 / p s i$ \\
\hline$c_{t}$ & $1.80 \mathrm{E}-05$ & \\
\hline
\end{tabular}

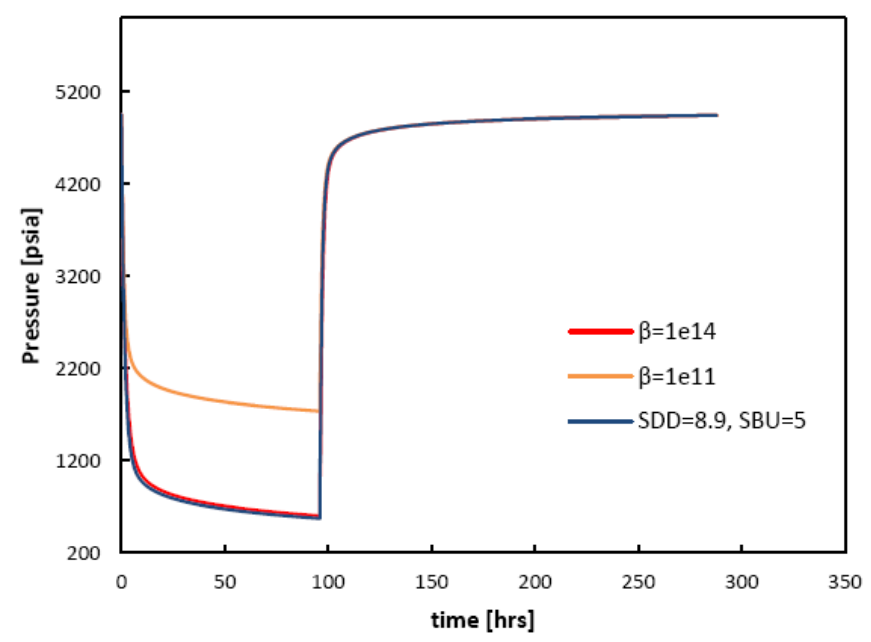

Figure6. Pressure Response for three cases

Figure shows the Horner plot for the three cases. The analysis of the three cases are summarized in Table. The results show that $\beta$-factor can be modelled by using skin factors. The application of Horner's technique inherently assumes superposition. The deeper point here being the fact that even though the problem is described by non-linear differential equations, superposition is still valid for larger values of time, because at longer times the problem essentially becomes linear.

Table2. Analysis results for the Horner plot for the three cases

\begin{tabular}{|c|c|c|c|}
\hline Case & $\boldsymbol{\beta}=\mathbf{1 e 1 4}[\mathbf{1} / \mathbf{f t}]$ & $\boldsymbol{\beta}=\mathbf{1 e 1 1}[\mathbf{1} / \mathbf{f t}]$ & $\mathbf{S D D = 8 . 9 ,} \mathbf{S B U = 5}$ \\
\hline slope [psi/cycle] & 338.2 & 338.2 & 338.2 \\
\hline $\mathbf{p}_{\mathbf{w f}}[\mathbf{p s i a}]$ & 599.9 & 1734.9 & 590.1 \\
\hline $\mathbf{p}_{\mathbf{1 h r}}[\mathbf{p s i a}]$ & 4328.0 & 4328.0 & 4328.0 \\
\hline $\mathbf{k}[\mathbf{m D}]$ & 1.0 & 1.0 & 1.0 \\
\hline skin & 8.9 & 5.1 & 8.9 \\
\hline
\end{tabular}

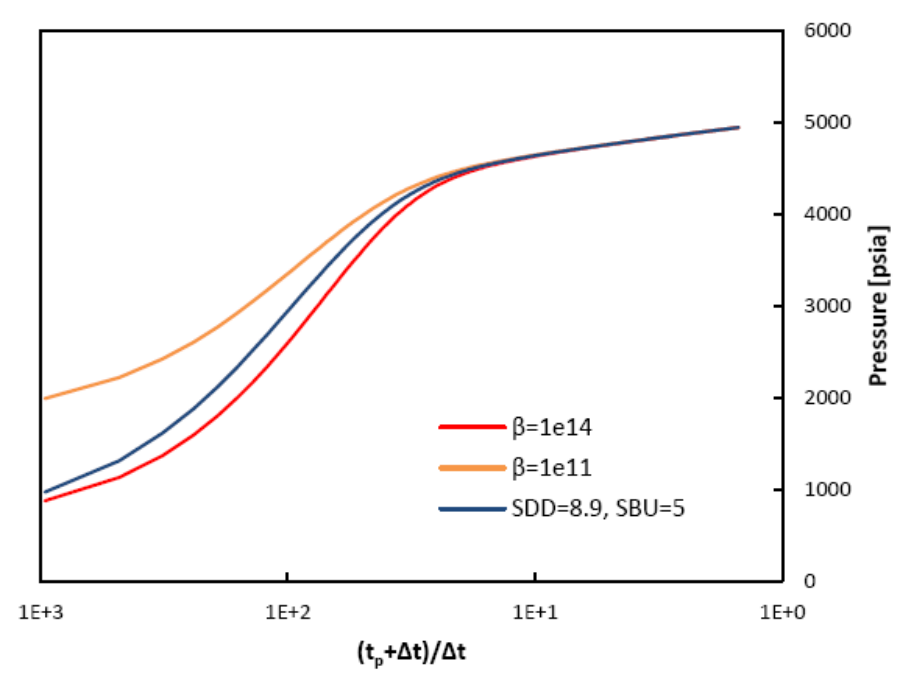

Figure7. Horner Plot for three cases 
Therefore, it can be concluded that since the problem is linear, superposition is still valid. Moreover, the analysis of the problem suggests that using skin factors to model $\beta$-factors is valid within engineering accuracy.

\section{Conclusion}

It is known that skin from Horner plot is the skin prevalent during drawdown regime. Three cases with different $\beta$-factors were analyzed using Horner plot. And since Horner plot assumes superposition, even though the problem is described by non-linear differential equations, superposition is still valid for larger values of time, which essentially renders the problem to become linear.

Moreover, it was observed that using skin is an adequate model to account for the non-Darcy $\beta$-factor for pressure transient problems.

\section{NOMENCLATURE}

$C \quad$ Wellbore storage coefficient, $\mathrm{m} 3 / \mathrm{Pa}$

$C_{D} \quad$ Dimensionless wellbore storage coefficient, non-Newtonian fluid

$c_{t} \quad$ Total compressibility, $\mathrm{Pa}^{-1}$

$D \quad$ Rate dependent skin constant, Day/MMscf

$h \quad$ Formation thickness, $m$

$k \quad$ Permeability, $\mathrm{m}^{2}\left[1 \mathrm{md}=9.86923 \times 10^{-15} \mathrm{~m}^{2}\right]$

$k_{a p p} \quad$ apparent rate-dependent permeability, $\mathrm{m}^{2}$

$k_{d} \quad$ constant Darcy permeability, $\mathrm{m}^{2}$

$k_{\min } \quad$ minimum permeability at high rate, $\mathrm{m}^{2}$

$p \quad$ Pressure, $\mathrm{Pa}$

$p_{D} \quad$ Dimensionless pressure

$p_{D}$, Dimensionless pressure derivative

$p_{i} \quad$ Initial Pressure, $\mathrm{Pa}$

$p_{r} \quad$ Reservoir pressure, $\mathrm{Pa}[1 \mathrm{psi}=6894.76 \mathrm{~Pa}]$

$p_{w} \quad$ Measured pressure at wellbore, $\mathrm{Pa}[1 \mathrm{psi}=6894.76 \mathrm{~Pa}]$

$p_{w D} \quad$ Dimensionless pressure drop at wellbore

$p_{w f} \quad$ Flowing wellbore pressure, $\mathrm{Pa}[1 \mathrm{psi}=6894.76 \mathrm{~Pa}]$

$q \quad$ Injection rate, $\mathrm{m}^{3} / \mathrm{s}\left[1 \mathrm{stb} / \mathrm{d}=1.84 \times 10^{-6}\right]$

$q_{g} \quad$ Gas flow rate, MMscfD

$r_{D} \quad$ Dimensionless radial distance

$R_{e} \quad$ Reynold's Number

$r_{w} \quad$ Wellbore radius, $\mathrm{m}$

$S$

$S_{B U}$

van Everdingen-Hurst skin factor

Skin during buildup

$s_{D D} \quad$ Skin during drawdown

$t \quad$ Time, $\mathrm{s}$

$T \quad$ Reservoir Temperature, $\mathrm{R}$

$t_{D} \quad$ Dimensionless time

$t_{p} \quad$ Producing time, hrs

$t_{p D} \quad$ Dimensionless production time

$U \quad$ Unit step-function described by Figure 1

USL Unit Slope Line

$v \quad$ Velocity, $\mathrm{m} / \mathrm{s}$

$\beta \quad$ Non-Darcy flow coefficient, $1 / \mathrm{ft}$

$\Delta t \quad$ Shut-in time, hrs

$\lambda \quad$ Mobility, $\mathrm{m}^{2} / \mathrm{Pa} . \mathrm{s}[\lambda=k / \mu]$

$\mu \quad$ Viscosity, Pa.s $[1 \mathrm{cp}=0.001$ Pa.s $]$

$\rho \quad$ Fluid density, $\mathrm{kg} / \mathrm{m}^{3}\left[1 \mathrm{lb} / \mathrm{ft}^{3}=16 \mathrm{~kg} / \mathrm{m}^{3}\right]$

$\tau \quad$ Alternate time, $\mathrm{s}$

$\varphi \quad$ Porosity

$\psi \quad$ Real gas pseudo-pressure, $\mathrm{psi}^{2} / \mathrm{cp}$ 


\section{REFERENCES}

[1] Ahmed, N. (1967). Physical Properties of Porous Medium Affecting Laminar and Turbulent Flow of Water. Fort Collins, Colorado: PhD Dissertation, Colorado State University.

[2] Barree, R. D., \& Conway, M. W. (2004). Beyond beta factors: a complete model for Darcy, Forchheimer, and trans-Forchheimer flow in porous media. SPE Annual Technical Conference and Exhibition. Houston: SPE. doi:10.2118/89325-MS

[3] Barree, R. D., \& Conway, M. W. (2005, August). Reply to Discussion of "Beyond Beta Factors: A Complete Model for Darcy, Forchheimer, and Trans-Forchheimer Flow in Porous Media". Journal of Petroleum Technology, 57(8), 73. doi:10.2118/0805-0073-JPT

[4] Blake, F. C. (1922). The resistance of packing to fluid flow. Transactions American Institute of Chemical Engineers, 4, pp. 415-421.

[5] Brownell, L. E., Gami, D. C., Miller, R. A., \& Nekarvis, W. F. (1956). Pressure Drop through Porous Media. AIChE Journal, 2(1), 79-81. doi:10.1002/aic.690020117

[6] Fancher, G. H., Lewis, J. A., \& Barnes, K. B. (1932). Some Physical Characteristics of Oil Sands. Pennsylvania State College Bulletin - Mineral Industries Experiment Station, 65.

[7] Forchheimer, P. (1901). Wasserbewegung durch Boden. Zeits. V. Deutsch. Ing., 45, 1781.

[8] Forchheimer, P. (1930). Hydraulik (third ed.). Leipzig: Druck und Verlag Von B.G. Teubner.

[9] Frederick Jr., D., \& Graves, R. M. (1994). New Correlations To Predict Non-Darcy Flow Coefficients at Immobile and Mobile Water Saturation. SPE Annual Technical Conference and Exhibition (p. 77). New Orleans: SPE. doi:10.2118/28451-MS

[10] Geertsma, J. (1974, October). Estimating the Coefficient of Inertial Resistance in Fluid Flow Through Porous Media. SPEJ, 14(5), 445-450. doi:10.2118/4706-PA

[11] Jones, S. C. (1987). Using the Inertial Coefficient, B, To Characterize Heterogeneity in Reservoir Rock. SPE Annual Technical Conference and Exhibition. Dallas TX: SPE. doi:10.2118/16949-MS

[12] Kim, B. Y. (1985). The Resistance to Flow in Simple and Complex Porous Media Whose Matrices are Composed of Spheres. Honolulu, Hawaii: MSc thesis, University of Hawaii at Manoa.

[13] Li, D., \& Engler, T. W. (2001). Literature Review on Correlations of the Non-Darcy Coefficient. SPE Permian Basin Oil and Gas Recovery Conference. Midland, TX: SPE. doi:10.2118/70015MS

[14] Lindquist, E. (1933). On the flow of water through porous soil. Premier Congres des grands barrages, (pp. 81-101). Stockholm.

[15] Mobasheri, F., \& Todd, D. K. (1963). Investigation of the hydraulics of flow near recharge wells. University of California. Hydraulic Laboratory.

[16] Pascal, H., Ronald, G. Q., \& Kingston, D. J. (1980). Analysis Of Vertical Fracture Length And Non-Darcy Flow Coefficient Using Variable Rate Tests. SPE Annual Technical Conference and Exhibition. Dallas TX: SPE. doi:10.2118/9348-MS

[17] Siddiqui, F., Soliman, M. Y., Waylon, H., \& Akif, I. (2016, 1). Pre-Darcy Flow Revisited under Experimental Investigation. Journal of Analytical Science and Technology. doi:10.1186/s40543015-0081-2

[18] Soliman, M. Y., Hedayati, S., \& Daneshi, T. (1991). Analysis of Buildup and Drawdown Tests With Variable Wellbore Storage and Skin. SPE Production Operations Symposium. Oklahoma, OK. doi:10.2118/21708-MS

[19] Sunada, D. K. (1965). Laminar and Turbulent Flow of Water through Homogeneous Porous Media. Berkeley, California: PhD dissertation, University of California at Berkeley.

[20] Wattenbarger, R. A., \& Ramey, H. J. (1968, August). Gas Well Testing With Turbulence, Damage and Wellbore Storage. JPT, 20(8), 877-887. doi:10.2118/1835-PA 